\title{
Base-free direct synthesis of alkynylphosphonates from alkynes and $H$-phosphonates catalyzed by $\mathrm{Cu}_{2} \mathrm{O}$.
}

\author{
Yanina Moglie, ${ }^{\mathrm{a},{ }^{*}}$ Evangelina Mascaró, ${ }^{\mathrm{a}}$ Victoria Gutierrez, ${ }^{\mathrm{a}}$ Francisco Alonso, ${ }^{\mathrm{b}}$ and Gabriel Radivoy. ${ }^{\mathrm{a}, *}$ \\ a Instituto de Química del Sur, INQUISUR (CONICET-UNS), Departamento de Química, Universidad Nacional del Sur, \\ Avenida Alem 1253, 8000 Bahía Blanca, Argentina. \\ ${ }^{\mathrm{b}}$ Instituto de Síntesis Orgánica (ISO) and Departamento de Química Orgánica, Facultad de Ciencias, Universidad de Alican- \\ te, Apdo. 99, 03080, Alicante, Spain.
}

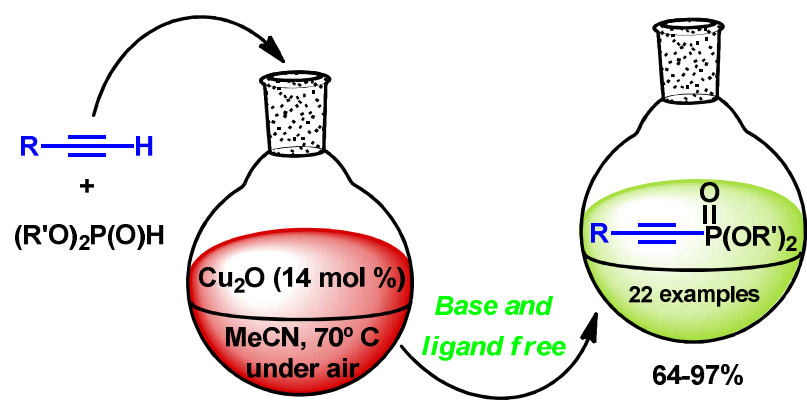

\begin{abstract}
A simple and mild methodology for the direct synthesis of alkynylphosphonates is presented. The reaction of a variety of terminal alkynes with dialkyl phosphites in the presence $\mathrm{Cu}_{2} \mathrm{O}(14 \mathrm{~mol} \%)$ led to the formation of the corresponding alkynylphosphonates in good to excellent yields. Reactions are performed under air, in acetonitrile as solvent, and in the absence of base or ligand additives. This new methodology is compatible with the presence of a wide variety of functional groups on the starting alkynes and can be scaled-up to a gram-scale.
\end{abstract}

\section{INTRODUCTION}

Alkynylphosphonates are one of the most valuable phosphorus derivatives in synthetic organic chemistry. The increasing interest in these compounds is mainly related to the wide presence of the phosphonic functionality (acid or ester) in many natural or synthetic bioactive compounds. ${ }^{1}$ On the other hand, the carbon-carbon triple bond provides great synthetic versatility to these phosphorus derivatives, allowing a variety of subsequent structural modifications such as addition or cycloaddition reactions, ${ }^{2}$ including "click" reactions for the synthesis of triazolyl-functionalized phosphonates with distinct biological activity. ${ }^{3}$

Traditional synthesis of alkynylphosphonates, by reacting $(\mathrm{RO})_{2} \mathrm{P}(\mathrm{O}) \mathrm{Cl}$ as phosphorus electrophiles with $\mathrm{Li}$ or Mg acetilydes, ${ }^{4}$ suffers from several drawbacks such as the use of air- and humidity-sensitive chemicals, and lack of functional group tolerance. Consequently, alternative methods have been developed in recent years, most of them based on the use of transition metal catalysts. Many of the reported methodologies require the use of func- 
tionalized starting alkenes or alkynes, including 1,1 -dibromoalkenes, ${ }^{5}$ propiolic acids, ${ }^{6}$ metal acetylides, ${ }^{7}$ alkynyl sulfones, ${ }^{8}$ trialkylsilyl alkynes, ${ }^{9}$ and 4-aryl-2-methyl-3-butyn-2-ols. ${ }^{10}$ For technical and economic reasons, the direct synthesis of alkynylphosphonates from commercial or readily available starting alkynes, is the most attractive way for the synthesis of these phosphorous compounds. In this regard, only two general direct synthetic methods have been published so far. In 2009, Han et al. ${ }^{11}$ reported an efficient procedure based on coppercatalyzed oxidative coupling of terminal alkynes and dialkyl phosphites, by using $\mathrm{CuI}$ as copper source and DMSO as solvent, under dry air. It is noteworthy that the use of MeCN, THF, dioxane, $\mathrm{CH}_{2} \mathrm{Cl}_{2}$ or toluene as solvent, only produced trace amounts of the desired product. The proposed reaction mechanism involves the formation of copper acetylide species, so the use of a base is mandatory for the deprotonation of the starting alkyne. In 2011, Wang and coworkers, ${ }^{12}$ reported the direct synthesis of alkynylphosphonates by using a silica-supported carbene- $\mathrm{Cu}(\mathrm{II})$ catalyst. Even though the synthesis of the catalyst is not straightforward, the process is base-free but the catalyst could be recovered and reused. Here again, the choice of the reaction solvent was crucial for the cross-coupling to take place. Thus, the use of solvents different from DMSO (DMF, THF, toluene, $\mathrm{CH}_{3} \mathrm{OH}$ ), gave no reaction product. Very recently, the group of Han reported a $\mathrm{Pd}(\mathrm{II}) / \mathrm{Ag}(\mathrm{I})$-mediated method for the dehydrogenative coupling of terminal alkynes with different $\mathrm{P}(\mathrm{O})-\mathrm{H}$ compounds, ${ }^{13}$ but only one example for the synthesis of an alkynyl phosphonate was reported.

Owing to our recent interest in phosphorus chemistry, ${ }^{14}$ we have recently reported our findings on the direct synthesis of $\beta$-ketophosphonates and vinylphosphonates from alkynes or alkenes catalyzed by copper nanoparticles supported on $\mathrm{ZnO}^{14 \mathrm{c}}$ In the course of further studies aimed at exploring other synthetically useful applications of this methodology, we found that commercial $\mathrm{Cu}_{2} \mathrm{O}$ was able to catalyze the direct cross-coupling between phenylacetylene and $\mathrm{HP}(\mathrm{O})(\mathrm{OEt})_{2}$, leading to the corresponding alkynylphosphonate product in good yield, together with minor amounts of 1,4-diphenyl-1,3-butadyine coming from the homocoupling of the starting alkyne (Glaser dimerization). The reaction was performed in the presence of $7 \mathrm{~mol} \%$ of $\mathrm{Cu}_{2} \mathrm{O}$, in $\mathrm{MeCN}$ as solvent, under air, and in the absence of any base additive or ligand (Scheme 1).

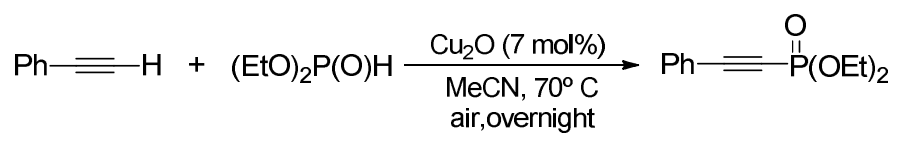

Scheme 1. $\mathrm{Cu}_{2} \mathrm{O}$-catalyzed cross-coupling of phenylacetylene with diethyl phosphite. 
We want to present herein a new simple and mild protocol for the direct synthesis of alkynylphosphonates from terminal alkynes and dialkyl phosphites catalyzed by copper(I) oxide.

\section{RESULTS AND DISCUSSION}

We started our study by optimizing the reaction conditions, using phenylacetylene (1a) as model substrate. Thus, the alkyne $(0.5 \mathrm{mmol})$ was added to a brownish-red suspension of $\mathrm{HP}(\mathrm{O})(\mathrm{OEt})_{2}(0.7 \mathrm{mmol})$ and $\mathrm{Cu}_{2} \mathrm{O}(5$ $\mathrm{mg}, 0.035 \mathrm{mmol})$ in $\mathrm{MeCN}(2 \mathrm{~mL})$, and stirred at $70{ }^{\circ} \mathrm{C}$ overnight under air. The reaction mixture turned to a clear pale-green solution and, after chromatographic purification, gave the alkynylphosphonate $\mathbf{2 a}$ in $77 \%$ yield, together with $15 \%$ of 1,4-diphenyl-1,3-butadyine and minor amounts of $(\mathrm{EtO})_{2} \mathrm{P}(\mathrm{O}) \mathrm{OH}$ and $\left[(\mathrm{EtO})_{2} \mathrm{P}(\mathrm{O})\right]_{2} \mathrm{O}$. As shown in Table 1, the choice of the solvent demonstrated to be important for the cross-coupling reaction but not as crucial as for the methodologies previously reported by Han and Wang. ${ }^{11,12}$ Thus, the use of polar protic solvents such as $\mathrm{H}_{2} \mathrm{O}$ or $\mathrm{CH}_{3} \mathrm{OH}$, gave no conversion to the desired alkynylphosphonate, whereas the use of DMSO or THF led to the corresponding cross-coupling product (Table 1, entries 2-5), albeit in significantly lower yield compared to that obtained by using $\mathrm{MeCN}$ as solvent.

Table 1. Screening of reaction conditions $\mathrm{s}^{\mathrm{a}}$

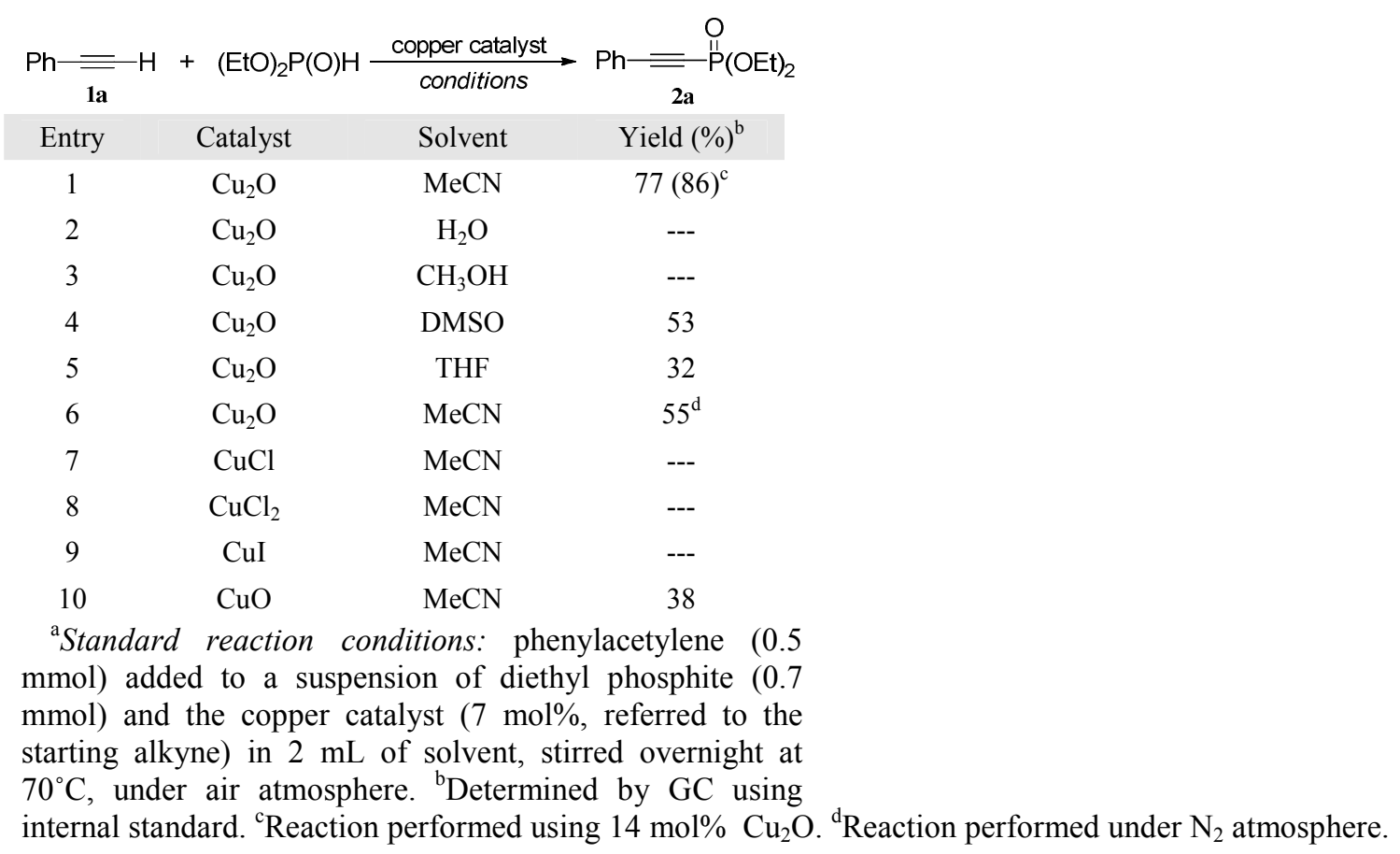

internal standard. ${ }^{\mathrm{c}}$ Reaction performed using $14 \mathrm{~mol}_{0} \mathrm{Cu}_{2} \mathrm{O}$. ${ }^{\mathrm{d}}$ Reaction performed under $\mathrm{N}_{2}$ atmosphere. 
As expected, the reaction carried out under nitrogen atmosphere allowed to minimize the formation of the diyne by-product, but the conversion to the desired alkynylphosphonate was lower than that obtained by working under air (Table 1, entry 6). Different copper sources were then tested as catalysts. As shown in Table 1 (entries 7-10) $\mathrm{CuCl}, \mathrm{CuCl}_{2}$ and $\mathrm{CuI}$ failed in catalyzing the cross-coupling reaction, whereas $\mathrm{CuO}$ gave only $38 \%$ of conversion into the alkynylphosphonate product. We then optimized the catalyst loading and found that by doubling the amount of $\mathrm{Cu}_{2} \mathrm{O}(10 \mathrm{mg}, 14 \mathrm{~mol} \%$ ) the reaction yield was increased from $77 \%$ to $86 \%$ (Table 1 , entry 1$)$. A further increase in the amount of $\mathrm{Cu}_{2} \mathrm{O}$ did not improve the reaction yield. Then, the influence of the order of addition of reactants was studied. Thus, when the synthesis of $\mathbf{2 a}$ was carried out by adding diethyl phosphite to a suspension of $\mathbf{1 a}$ and $\mathrm{Cu}_{2} \mathrm{O}$ in $\mathrm{MeCN}$, only $57 \%$ conversion into $2 \mathbf{a}$ was obtained.

With the optimized conditions in hand, we then studied the substrate scope of our methodology by reacting a wide range of alkynes bearing different functional groups. As shown in Table 2, the $\mathrm{Cu}_{2} \mathrm{O}$-catalyzed crosscoupling reaction proved to be compatible with the presence of a great variety of functional groups in the starting alkyne. Aromatic substrates gave the corresponding alkynylphosphonate products in good to excellent yields, irrespective of the electronic properties of the substituents attached to the aromatic ring (Table 2, entries 2-7). Aliphatic alkynes, including those substituted with other functional groups (halogen, nitrile, hydroxyl, ketal, imide) also gave the desired alkynylphosphonate products in good yields (Table 2, entries 8-13, 19). It is worth noting that the reaction of 1,8-nonadiyne with an excess of $\mathrm{HP}(\mathrm{O})(\mathrm{OEt})_{2}$ gave $70 \%$ of the corresponding monophosphonated product (Table 2, entry 14). Except for alkynes containing carboxylic acids or primary amino groups, which gave no coupling products, our methodology could be also applied to ynones, propiolates and enynes (Table 2, entries 15-18). Interestingly, ethynylestradiol could be converted into its alkynylphosphonate analogue in good yield (Table 2, entry 20). On the other hand, dibutyl- and dimethyl phosphite could also be used as starting $H$-phosphonates giving the corresponding alkynylphosphonates in good yield (Table 2, entry 1). Unfortunately, the methodology could not be extended to the synthesis of other alkynyl phosphorus derivatives such as alkynylphosphine oxides and alkynylphosphonites. The reaction of di-phenylphosphine oxide with phenylacetylene under the optimized conditions, led to a low conversion of the starting alkyne (30\%) giving minor amounts of the corresponding alkynylphosphine oxide (8\%), together with a mixture of di-phenylvinylphosphine oxide products, 
probably coming from the hydrophosphorylation of phenylacetylene. On the other hand, the reaction of dibutylphosphonite with phenylacetylene gave no conversion of the starting alkyne.

Taking into account that gram-scale synthesis of alkynylphosphonates could surely be of interest in various fields of application, we decided to study whether our methodology could be scaled-up. Thus, the synthesis of alkynylphosphonate 2a $\left(\mathrm{R}^{\prime}=\mathrm{Et}\right)$ was carried out by using a twelve-fold amount of the reactants, giving the corresponding cross-coupling product in $75 \%$ yield $(1.07 \mathrm{~g})$ after $24 \mathrm{~h}$ of reaction time (Table 2 , entry 1 , footnote c).

Although the exact mechanistic pathway is difficult to ascertain at this stage, the fact that the cross-coupling reaction takes place in the absence of any added base, led us to think about a plausible reaction mechanism different from that proposed by Han et al., ${ }^{11}$ i.e. copper acetylide species should not be the main reaction intermediates under our reaction conditions. Moreover, it is striking to note that $\mathrm{Han}$ and co-workers also tested $\mathrm{Cu}_{2} \mathrm{O}$ as catalyst, and a conversion of only $7 \%$ to the cross-coupling product was observed. This could indicate that the formation of a copper acetylide is negligible in the presence of $\mathrm{Cu}_{2} \mathrm{O}$ and DMSO as solvent, even when $\mathrm{K}_{2} \mathrm{CO}_{3}$ is being used as base.

Table 2. $\mathrm{Cu}_{2} \mathrm{O}$-catalyzed direct synthesis of alkynylphosphonates ${ }^{\mathrm{a}}$

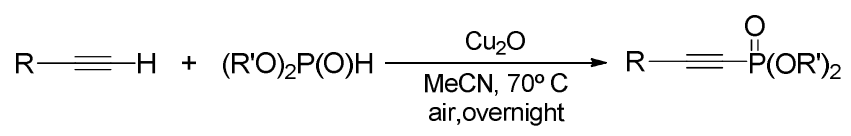

$\begin{array}{ll}\text { Entry } & \begin{array}{l}\text { Yield (\%) } \\ \mathrm{R}^{\prime}=\mathrm{Et}: 86^{\mathrm{c}}\end{array} \\ \mathrm{R}^{\prime}=\mathrm{Me}: 90 \\ \mathrm{R}^{\prime}=\mathrm{Bu}: 83\end{array}$




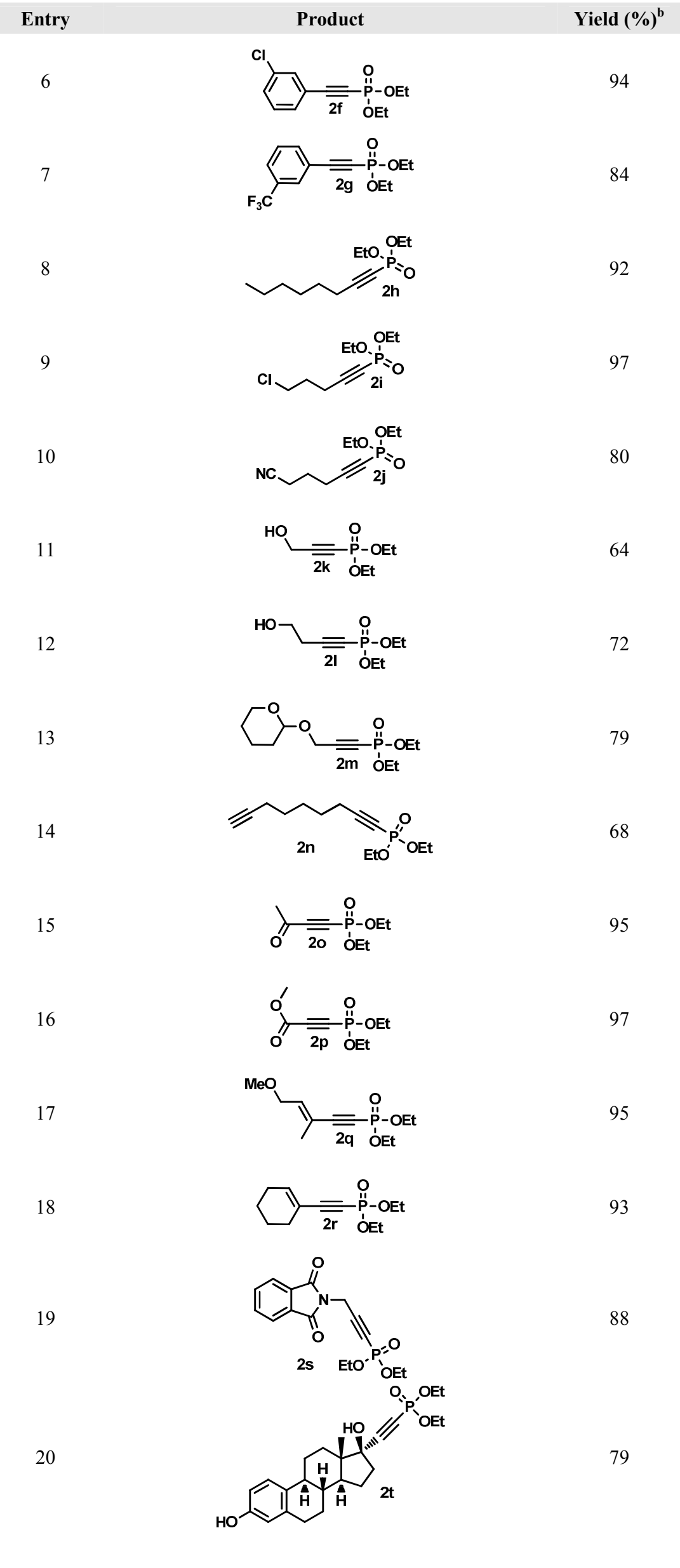


${ }^{a}$ Reaction conditions: alkyne $(0.5 \mathrm{mmol})$ added to a suspension of $\mathrm{H}$ phosphonate $(0.7 \mathrm{mmol})$ and $\mathrm{Cu}_{2} \mathrm{O}(14 \mathrm{~mol} \%)$ in $\mathrm{MeCN}(2 \mathrm{~mL})$, stirred overnight at $70^{\circ} \mathrm{C}$ under air. ${ }^{\mathrm{b}}$ Isolated yield after column chromatography. ${ }^{\mathrm{c}} \mathrm{Scaled}-\mathrm{up}$ synthesis of $\mathbf{2 a}(\mathrm{R}=\mathrm{Et})$ : phenylacetylene $(6 \mathrm{mmol}),(\mathrm{EtO}){ }_{2} \mathrm{P}(\mathrm{O}) \mathrm{H}$ ( $8.4 \mathrm{mmol}), \mathrm{Cu}_{2} \mathrm{O}(14 \mathrm{~mol} \%)$ in $\mathrm{MeCN}(20 \mathrm{~mL}), 24 \mathrm{~h}, 75 \%$ yield.

To confirm our assumptions, we carried out some additional experiments. Initially, the reaction by using copper phenylacetylide as starting material, both in the presence or absence of $\mathrm{Cu}_{2} \mathrm{O}$, gave no conversion into the alkynylphosphonate 2a. In another experiment, when 1-octyne was reacted with diethyl phosphite under the optimized conditions, in the presence of $\mathrm{K}_{2} \mathrm{CO}_{3}$ or $\mathrm{Et}_{3} \mathrm{~N}$ as base, a notably lower conversion to the alkynyl phosphonate $\mathbf{2 h}$ was obtained ( $25 \%$ and $40 \%$, respectively) compared to that of the reaction performed in the absence of any added base $(92 \%$, Table 2 , entry 8$)$. Finally, the synthesis of $\mathbf{2 a}\left(\mathrm{R}^{\prime}=\mathrm{Et}\right)$ under the optimized conditions, was not affected by the addition of TEMPO as radical scavenger.

In the light of our experimental results, and those previously reported by Han and coworkers, ${ }^{11}$ it could be inferred that the cross-coupling reaction might take place through different pathways depending on both the source of copper and the solvent used. Moreover, the experimental results observed when using different solvents for the coupling reaction (Table 1), could be related to the ability of the solvent to act as a ligand for copper, thus possibly favouring an oxidative addition-reductive elimination process under our reaction conditions. Even though the specific oxidation states and structures of the copper intermediates remain unclear, we speculate that a $\mathrm{Cu}(\mathrm{I}) / \mathrm{Cu}(\mathrm{III})$ redox couple is required since the efficiency of the reaction decreased in the absence of oxygen. ${ }^{15}$ Based on these premises, a reaction mechanism has been proposed including: (a) copper activation by the solvent to form $\mathrm{MeCN}-\mathrm{Cu}(\mathrm{I})$ species, (b) reaction of $\mathrm{Cu}(\mathrm{I})$ with the dialkyl phosphite leading to copper(I) dialkyl phosphite, (c) reaction of the latter with the terminal alkyne in the presence of oxygen to provide a $\mathrm{Cu}(\mathrm{II})$ intermediate, (d) oxidation of copper(II) to copper(III) and (e) subsequent reductive elimination ${ }^{16}$ to produce the alkynyl phosphonate with the concomitant regeneration of copper(I) (Scheme 2). 

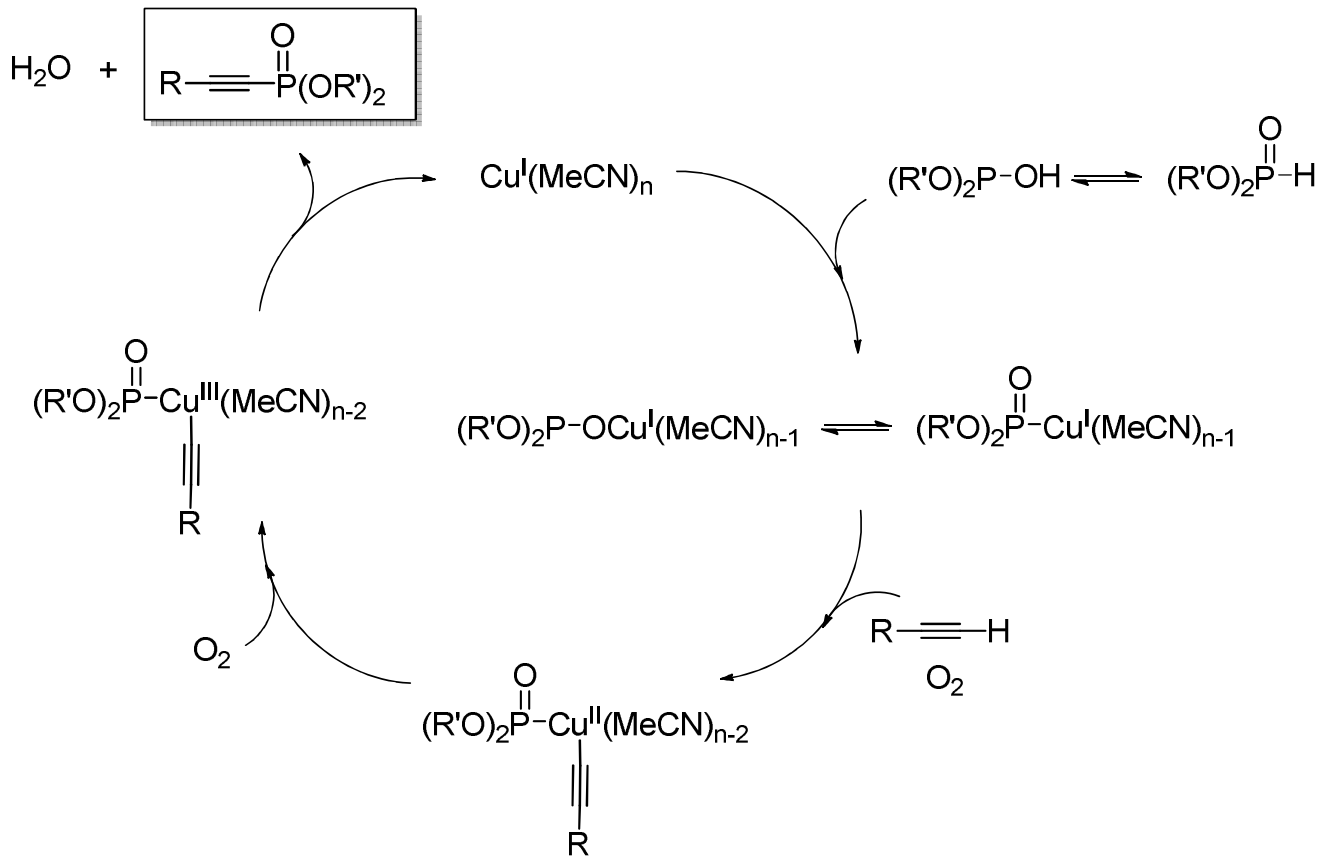

Scheme 2. Proposed reaction pathway.

\section{CONCLUSION}

In conclusion, we have described a one pot methodology for the base-free direct synthesis of alkynylphosphonates from commercial or readily available terminal alkynes, catalyzed by $\mathrm{Cu}_{2} \mathrm{O}$ under air atmosphere. The new methodology showed to be compatible with a wide variety of functional groups in the starting alkynes and could be scaled-up to a gram-scale. Although the exact mechanistic pathway remained to be studied more in detail, based on our experimental observations and previous results reported by other authors, we assume that an oxidative addition-reductive elimination process could take place under our reaction conditions. This new methodology is simple, efficient and allows the direct synthesis of alkynylphosphonates with high atom economy. Further mechanistic studies, including computational analysis using DFT, are now in progress.

\section{EXPERIMENTAL}

\section{General}

All starting materials were of the best available grade and were used without further purification. Column chromatography was performed using silica gel $60(0.040-0.063 \mu \mathrm{m}, 240-400$ mesh). Reactions were monitored by 
thin-layer chromatography on silica gel plates (60F-254) visualized under UV light and/or using 5\% $\mathrm{KMnO}_{4}$ in water.

Nuclear magnetic resonance (NMR) spectra were recorded at $300 \mathrm{MHz}$ for ${ }^{1} \mathrm{H} \mathrm{NMR} ; 75 \mathrm{MHz}$ for ${ }^{13} \mathrm{C}$ NMR and $121 \mathrm{MHz}$ for ${ }^{31} \mathrm{P} \mathrm{MNR}$; using $\mathrm{CDCl}_{3}$ as the solvent and tetramethylsilane (TMS) as internal reference. Chemical shifts $(\delta)$ are reported in parts per million $(\mathrm{ppm})$ from tetramethylsilane (TMS) using the residual solvent resonance $\left(\mathrm{CDCl}_{3}: 7.26 \mathrm{ppm}\right.$ for ${ }^{1} \mathrm{H}$ NMR, $77.16 \mathrm{ppm}$ for $\left.{ }^{13} \mathrm{C} \mathrm{NMR}\right)$. Multiplicities are abbreviated as follows: $\mathrm{s}=$ singlet, $\mathrm{d}=$ doublet, $\mathrm{t}=$ triplet, $\mathrm{q}=$ quartet, $\mathrm{m}=$ multiplet; brs = broad signal). Coupling constants $(J)$ were reported in $\mathrm{Hz}$.

Mass spectra (EI) were obtained at $70 \mathrm{eV}$ on a GC/MS instrument equipped with a selective mass detector. Infrared (FT-IR) spectra were obtained on a spectrophotometer equipped with an ATR component. Melting points are uncorrected. The purity of volatile compounds and the chromatographic analyses (GC) were determined with a instrument equipped with a flame-ionisation detector and a $30 \mathrm{~m}$ column $(0.25 \mathrm{~mm}, 0.25 \mu \mathrm{m})$, using nitrogen as carrier gas. High resolution mass spectra were recorded on a spectrometer equipped with a Orbitrap XL mass analyzer, (for EI) and a MAT 95 (for ESI).

\section{General procedure for the synthesis of alkynylphosphonates 2.}

The dialkyl phosphite $(0.7 \mathrm{mmol})$ was added to a brownish-red suspension suspension of $\mathrm{Cu}_{2} \mathrm{O}(10 \mathrm{mg}, 0.07$ $\mathrm{mmol})$ in acetonitrile $(2 \mathrm{~mL})$. Then, the corresponding alkyne $(0.5 \mathrm{mmol})$ was added and the reaction mixture was warmed to $70^{\circ} \mathrm{C}$ and stirred overnight under air. The reaction mixture turned a pale-green clear solution. The solvent was evaporated in vacuo and the crude product was purified by flash column chromatography (silica gel, hexane/AcOEt).

3. Gram-scale synthesis of diethyl (phenylethynyl)phosphonate. Diethyl phosphite ( $8.4 \mathrm{mmol}, 1082 \mu \mathrm{L})$ was added to a suspension of the $\mathrm{Cu}_{2} \mathrm{O}(60 \mathrm{mg}, 14 \mathrm{~mol} \%)$ in $\mathrm{MeCN}(20 \mathrm{~mL})$. Then, phenylacetylene $(6.0 \mathrm{mmol}, 660$ $\mu \mathrm{L}$ ) was added and the reaction mixture was warmed to $70{ }^{\circ} \mathrm{C}$ overnight under air atmosphere. The solvent was removed in vacuo and the product was purified by flash column chromatography (hexane-EtOAc, 4:6) to give diethyl (phenylethynyl)phosphonate in 75\% yield (1.07 g).

\section{Compound characterization data.}


All known compounds were characterized by comparison of their physical and spectroscopic data with those described in the literature. For new compounds, copies of ${ }^{1} \mathrm{H}-,{ }^{13} \mathrm{C}$ - and ${ }^{31} \mathrm{P}-\mathrm{NMR}$ graphical spectra are also provided (see Supporting Information).

Diethyl (phenylethynyl)phosphonate $(2 \mathbf{a}, \mathbf{R}=\mathbf{E t})^{17}$ : Yellow oil. $102.4 \mathrm{mg}, 86 \%$ yield. ${ }^{1} \mathrm{H}$ NMR $(300 \mathrm{MHz}$, $\left.\mathrm{CDCl}_{3}\right) \delta 7.61-7.53(\mathrm{~m}, 2 \mathrm{H}), 7.49-7.33(\mathrm{~m}, 3 \mathrm{H}), 4.31-4.17(\mathrm{~m}, 4 \mathrm{H}), 1.41(\mathrm{t}, J=7.1 \mathrm{~Hz}, 6 \mathrm{H}) .{ }^{13} \mathrm{C} \mathrm{NMR}(75$ $\left.\mathrm{MHz}, \mathrm{CDCl}_{3}\right) \delta 132.7(\mathrm{~d}, J=2.5 \mathrm{~Hz}), 130.8,128.7,119.7(\mathrm{~d}, J=5.7 \mathrm{~Hz}), 99.2(\mathrm{~d}, J=53.0 \mathrm{~Hz}), 78.5(\mathrm{~d}, J=300.0$ $\mathrm{Hz}), 63.4(\mathrm{~d}, J=5.5 \mathrm{~Hz}), 16.2(\mathrm{~d}, J=7.0 \mathrm{~Hz}) .{ }^{31} \mathrm{P} \mathrm{NMR}\left(121 \mathrm{MHz}, \mathrm{CDCl}_{3}\right) \delta-5.9 \mathrm{ppm}$.

Dimethyl (phenylethynyl)phosphonate (2a, $\mathbf{R}=\mathbf{M e})^{18}$ : Colorless oil. $87.2 \mathrm{mg}, 83 \%$ yield. ${ }^{1} \mathrm{H}$ NMR $(300 \mathrm{MHz}$, $\left.\mathrm{CDCl}_{3}\right) \delta 7.55-7.48(\mathrm{~m}, 2 \mathrm{H}), 7.44-7.28(\mathrm{~m}, 3 \mathrm{H}), 3.79(\mathrm{~d}, J=12.3 \mathrm{~Hz}, 6 \mathrm{H}) .{ }^{13} \mathrm{C} \mathrm{NMR}\left(75 \mathrm{MHz}, \mathrm{CDCl}_{3}\right) \delta$ $132.8(\mathrm{~d}, J=2.5 \mathrm{~Hz}), 131.0,128.7,119.4(\mathrm{~d}, J=5.7 \mathrm{~Hz}), 100.2(\mathrm{~d}, J=53.4 \mathrm{~Hz}), 76.9(\mathrm{~d}, J=303.3 \mathrm{~Hz}), 53.6(\mathrm{~d}, J$ $=5.5 \mathrm{~Hz}) .{ }^{31} \mathrm{P} \mathrm{NMR}\left(121 \mathrm{MHz}, \mathrm{CDCl}_{3}\right) \delta-2.7 \mathrm{ppm}$.

Dibutyl (phenylethynyl)phosphonate (2a, $\mathbf{R}=\mathbf{B u})^{19}$ : Colorless oil. $132.4 \mathrm{mg}, 90 \%$ yield. ${ }^{1} \mathrm{H}$ NMR $(300 \mathrm{MHz}$, $\left.\mathrm{CDCl}_{3}\right) \delta 7.60-7.53(\mathrm{~m}, 2 \mathrm{H}), 7.50-7.42(\mathrm{~m}, 1 \mathrm{H}), 7.40-7.34(\mathrm{~m}, 2 \mathrm{H}), 4.21-4.12(\mathrm{~m}, 4 \mathrm{H}), 1.79-1.67(\mathrm{~m}$, 4H), $1.53-1.39(\mathrm{~m}, 4 \mathrm{H}), 0.95(\mathrm{~d}, J=7.4 \mathrm{~Hz}, 6 \mathrm{H}) .{ }^{13} \mathrm{C} \mathrm{NMR}\left(75 \mathrm{MHz}, \mathrm{CDCl}_{3}\right) \delta 132.8(\mathrm{~d}, J=2.5 \mathrm{~Hz}), 130.8$, 128.7, $119.8(\mathrm{~d}, J=5.6 \mathrm{~Hz}), 99.2(\mathrm{~d}, J=52.7 \mathrm{~Hz}), 78.5(\mathrm{~d}, J=299.5 \mathrm{~Hz}), 67.1(\mathrm{~d}, J=5.9 \mathrm{~Hz}), 32.4(\mathrm{~d}, J=7.1$ $\mathrm{Hz}), 18.9,13.7 .{ }^{31} \mathrm{P} \mathrm{NMR}\left(121 \mathrm{MHz}, \mathrm{CDCl}_{3}\right) \delta-5.56 \mathrm{ppm}$.

Diethyl (p-methylphenyl)ethynyl phosphonate (2b) ${ }^{17}$ : Pale yellow oil. $112.2 \mathrm{mg}, 89 \%$ yield. ${ }^{1} \mathrm{H}$ NMR (300 $\left.\mathrm{MHz}, \mathrm{CDCl}_{3}\right) \delta 7.44(\mathrm{~d}, J=8.0 \mathrm{~Hz}, 2 \mathrm{H}), 7.16(\mathrm{~d}, J=7.9 \mathrm{~Hz}, 2 \mathrm{H}), 4.26-4.16(\mathrm{~m}, 4 \mathrm{H}), 2.36(\mathrm{~s}, 3 \mathrm{H}), 1.39(\mathrm{t}, J=$ $7.1 \mathrm{~Hz}, 6 \mathrm{H}) .{ }^{13} \mathrm{C} \mathrm{NMR}\left(75 \mathrm{MHz}, \mathrm{CDCl}_{3}\right) \delta 141.3,132.5(\mathrm{~d}, J=2.5 \mathrm{~Hz}), 129.3,116.4(\mathrm{~d}, J=5.6 \mathrm{~Hz}), 99.6(\mathrm{~d}, J=$ $53.3 \mathrm{~Hz}), 77.7(\mathrm{~d}, J=301.1 \mathrm{~Hz}), 63.2(\mathrm{~d}, J=5.5 \mathrm{~Hz}), 21.6,16.1(\mathrm{~d}, J=7.0 \mathrm{~Hz}) .{ }^{31} \mathrm{P} \mathrm{NMR}\left(121 \mathrm{MHz}, \mathrm{CDCl}_{3}\right) \delta-$ 5,7 ppm.

Diethyl (m-tolylethynyl)phosphonate (2c) $)^{20}$ : Yellow oil. $88.2 \mathrm{mg}, 70 \%$ yield. ${ }^{1} \mathrm{H}$ NMR $\left(300 \mathrm{MHz}, \mathrm{CDCl}_{3}\right) \delta$ $7.41-7.33(\mathrm{~m}, 2 \mathrm{H}), 7.30-7.22(\mathrm{~m}, 2 \mathrm{H}), 4.30-4.17(\mathrm{~m}, 4 \mathrm{H}), 2.35(\mathrm{~s}, 3 \mathrm{H}), 1.41(\mathrm{t}, J=7.1 \mathrm{~Hz}, 6 \mathrm{H}) .{ }^{13} \mathrm{C}$ NMR $\left(75 \mathrm{MHz}, \mathrm{CDCl}_{3}\right) \delta 138.5,133.2(\mathrm{~d}, J=2.5 \mathrm{~Hz}), 131.7,129.9(\mathrm{~d}, J=2.5 \mathrm{~Hz}), 128.6,119.4(\mathrm{~d}, J=5.6 \mathrm{~Hz}), 99.6$ $(\mathrm{d}, J=53.1 \mathrm{~Hz}), 78.0(\mathrm{~d}, J=300.7 \mathrm{~Hz}), 63.4(\mathrm{~d}, J=5.5 \mathrm{~Hz}), 21.2,16.2(\mathrm{~d}, J=7.0 \mathrm{~Hz}) .{ }^{31} \mathrm{P} \mathrm{NMR}(121 \mathrm{MHz}$, $\left.\mathrm{CDCl}_{3}\right) \delta-5.9 \mathrm{ppm}$.

Diethyl (4-methoxyphenyl)ethynylphosphonate (2d) ${ }^{17}$ : Yellow oil. $118.0 \mathrm{mg}, 88 \%$ yield. ${ }^{1} \mathrm{H}$ NMR (300 MHz, $\left.\mathrm{CDCl}_{3}\right) \delta 7.45(\mathrm{~d}, J=8.8 \mathrm{~Hz}, 2 \mathrm{H}), 6.82(\mathrm{~d}, J=8.9 \mathrm{~Hz}, 2 \mathrm{H}), 4.22-4.09(\mathrm{~m}, 4 \mathrm{H}), 3.77(\mathrm{~s}, 3 \mathrm{H}), 1.34(\mathrm{t}, J=7.110$ 
$\mathrm{Hz}, 6 \mathrm{H}) .{ }^{13} \mathrm{C} \mathrm{NMR}\left(75 \mathrm{MHz}, \mathrm{CDCl}_{3}\right) \delta 161.5,134.4(\mathrm{~d}, J=2.5 \mathrm{~Hz}), 114.3,111.3(\mathrm{~d}, J=5.7 \mathrm{~Hz}), 99.8(\mathrm{~d}, J=53.9$ $\mathrm{Hz}), 77.1(\mathrm{~d}, J=302.3 \mathrm{~Hz}), 63.1(\mathrm{~d}, J=5.5 \mathrm{~Hz}), 55.4,16.1(\mathrm{~d}, J=7.1 \mathrm{~Hz}) .{ }^{31} \mathrm{P}$ NMR $\left(121 \mathrm{MHz}, \mathrm{CDCl}_{3}\right) \delta-5.5$ ppm.

Diethyl (4-dimethylaminophenyl)ethynylphosphonate (2e) ${ }^{17}$ : maroon oil. $126.5 \mathrm{mg}, 90 \%$ yield. ${ }^{1} \mathrm{H}$ NMR (300 $\left.\mathrm{MHz}, \mathrm{CDCl}_{3}\right) \delta 7.35(\mathrm{~d}, J=8.9 \mathrm{~Hz}, 2 \mathrm{H}), 6.53(\mathrm{~d}, J=8.9 \mathrm{~Hz}, 2 \mathrm{H}), 4.20-4.07(\mathrm{~m}, 4 \mathrm{H}), 2.94(\mathrm{~s}, 6 \mathrm{H}), 1.32(\mathrm{t}, J=$ $7.1 \mathrm{~Hz}, 6 \mathrm{H}) .{ }^{13} \mathrm{C} \mathrm{NMR}\left(75 \mathrm{MHz}, \mathrm{CDCl}_{3}\right) \delta 151.6,134.2(\mathrm{~d}, J=2.5 \mathrm{~Hz}), 111.5,105.3(\mathrm{~d}, J=5.9 \mathrm{~Hz}), 102.3(\mathrm{~d}, J=$ $54.7 \mathrm{~Hz}), 76.4(\mathrm{~d}, J=305.1 \mathrm{~Hz}), 63.0(\mathrm{~d}, J=5.4 \mathrm{~Hz}), 40.1,16.3(\mathrm{~d}, J=7.1 \mathrm{~Hz}) .{ }^{31} \mathrm{P} \mathrm{NMR}\left(121 \mathrm{MHz}, \mathrm{CDCl}_{3}\right) \delta-$ $4.5 \mathrm{ppm}$.

Diethyl (3-chlorophenyl)ethynylphosphonate (2f): Pale yellow oil. 127.9 mg, 94\% yield. IR (neat): 2990, 2933, 2904, 2197, 1564, 1474, 1274, 1021, 976, 902, $796 \mathrm{~cm}^{-1} .{ }^{1} \mathrm{H}$ NMR (300 MHz, $\left.\mathrm{CDCl}_{3}\right) \delta 7.52(\mathrm{~s}, 1 \mathrm{H}), 7.47-7.37$ $(\mathrm{m}, 2 \mathrm{H}), 7.35-7.26(\mathrm{~m}, 1 \mathrm{H}), 4.26-4.16(\mathrm{~m}, 4 \mathrm{H}), 1.39(\mathrm{t}, J=7.1 \mathrm{~Hz}, 6 \mathrm{H}) .{ }^{13} \mathrm{C} \mathrm{NMR}\left(75 \mathrm{MHz}, \mathrm{CDCl}_{3}\right) \delta 134.6$, $132.4(\mathrm{~d}, J=2.6 \mathrm{~Hz}), 131.1,130.8(\mathrm{~d}, J=2.4 \mathrm{~Hz}), 130.0,121.4(\mathrm{~d}, J=5.7 \mathrm{~Hz}), 97.1(\mathrm{~d}, J=52.5 \mathrm{~Hz}), 79.7(\mathrm{~d}, J=$ $298.0 \mathrm{~Hz}), 63.5(\mathrm{~d}, J=5.6 \mathrm{~Hz}), 16.2(\mathrm{~d}, J=6.9 \mathrm{~Hz}) .{ }^{31} \mathrm{P}$ NMR $\left(121 \mathrm{MHz}, \mathrm{CDCl}_{3}\right) \delta-6,7 \mathrm{ppm} . \mathrm{MS}: m / z=272$ $\left(\mathrm{M}^{+}, 5 \%\right), 244$ (11), 229 (12), 216 (15), 209 (10), 201 (11), 199 (25), 165 (14), 164 (13), 163 (27), 162 (28), 155 (10), 154 (11), 145 (13), 139 (18), 138 (40), 137 (15), 136 (100), 129 (11), 128 (12), 123 (19), 107 (12), 101 (13), 89 (14), 75 (11). HRMS (EI) calcd for $\mathrm{C}_{12} \mathrm{H}_{14} \mathrm{ClO}_{3} \mathrm{P} 272.0369$, found 272.0374.

Diethyl (3-trifluoromethylphenyl)ethynylphosphonate (2g): Pale yellow oil. $128.5 \mathrm{mg}$, 84\% yield. IR (neat): 2990, 2937, 2908, 2197, 1319, 1270, 1139, 1029, 874, $775 \mathrm{~cm}^{-1} .{ }^{1} \mathrm{H}$ NMR $\left(300 \mathrm{MHz}, \mathrm{CDCl}_{3}\right) \delta 7.74-7.64(\mathrm{~m}$, 2H), $7.58-7.49(\mathrm{~m}, 2 \mathrm{H}), 4.29-4.13(\mathrm{~m}, 4 \mathrm{H}), 1.37(\mathrm{t}, J=7.1 \mathrm{~Hz}, 6 \mathrm{H}) .{ }^{13} \mathrm{C} \mathrm{NMR}\left(75 \mathrm{MHz}, \mathrm{CDCl}_{3}\right) \delta 135.2(\mathrm{~d}, J$ $=2.7 \mathrm{~Hz}), 132.7(\mathrm{qd}, J=31.1,1.7 \mathrm{~Hz}), 131.8,130.6,126.2(\mathrm{q}, J=5.0 \mathrm{~Hz}), 123.1(\mathrm{q}, J=273.6 \mathrm{~Hz}), 117.9-117.7$ (m), $94.1(\mathrm{~d}, J=51.8 \mathrm{~Hz}), 83.8(\mathrm{~d}, J=293.0 \mathrm{~Hz}), 63.6(\mathrm{~d}, J=5.7 \mathrm{~Hz}), 16.1(\mathrm{~d}, J=7.1 \mathrm{~Hz}) .{ }^{31} \mathrm{P}$ NMR $(121 \mathrm{MHz}$, $\left.\mathrm{CDCl}_{3}\right) \delta-7.3$ ppm. MS: $m / z=306\left(\mathrm{M}^{+}, 3 \%\right), 286(14), 279(11), 278$ (18), 277 (11), 250 (26), 248 (17), 239 (10), 238 (34), 237 (10), 233 (12), 214 (10), 213 (33), 211 (35), 210 (13), 209 (17), 197 (25), 196 (16), 186 (16), 185 (51), 177 (39), 174 (11), 171 (11), 170 (100), 169 (45), 159 (10), 158(12), 157 (38), 155(17), 151 (45), 138 (35), 137 (11), 128 (13), 120 (10), 119 (10), 116 (13), 99 (10), 88 (10). HRMS (EI) calcd for $\mathrm{C}_{13} \mathrm{H}_{14} \mathrm{~F}_{3} \mathrm{O}_{3} \mathrm{P}$ 306.0633, found 306.0638 .

Diethyl oct-1-yn-1-ylphosphonate (2h) ${ }^{21}$ : Yellow oil. $113.2 \mathrm{mg}, 92 \%$ yield. ${ }^{1} \mathrm{H}$ NMR $\left(300 \mathrm{MHz}, \mathrm{CDCl}_{3}\right) \delta 4.21$ $-4.08(\mathrm{~m}, 4 \mathrm{H}), 2.34(\mathrm{td}, J=7.1,4.4 \mathrm{~Hz}, 2 \mathrm{H}), 1.65-1.52(\mathrm{~m}, 2 \mathrm{H}), 1.43-1.25(\mathrm{~m}, 6 \mathrm{H}), 1.37(\mathrm{t}, J=7.1 \mathrm{~Hz}, 11$ 
$6 \mathrm{H}), 0.89(\mathrm{t}, J=6.8 \mathrm{~Hz}, 3 \mathrm{H}) .{ }^{13} \mathrm{C} \mathrm{NMR}\left(75 \mathrm{MHz}, \mathrm{CDCl}_{3}\right) \delta 103.4(\mathrm{~d}, J=53.2 \mathrm{~Hz}), 70.5(\mathrm{~d}, J=303.9 \mathrm{~Hz}), 63.0$ $(\mathrm{d}, J=5.5 \mathrm{~Hz}), 31.2,28.5,27.5(\mathrm{~d}, J=2.2 \mathrm{~Hz}), 22.5,19.3(\mathrm{~d}, J=4.5 \mathrm{~Hz}), 16.1(\mathrm{~d}, J=7.1 \mathrm{~Hz}), 14.0 .{ }^{31} \mathrm{P}$ NMR $\left(121 \mathrm{MHz}, \mathrm{CDCl}_{3}\right) \delta-6.1 \mathrm{ppm}$.

Diethyl (5-chloropent-1-yn-1-yl)phosphonate (2i): Colorless oil. 115.5 mg, 97\% yield. IR (neat): 2986, 2929, 2904, 2206, 1262, 1160, 1029, 972, 800, $771 \mathrm{~cm}^{-1} .{ }^{1} \mathrm{H}$ NMR (300 MHz, $\left.\mathrm{CDCl}_{3}\right) \delta 4.24-4.07(\mathrm{~m}, 4 \mathrm{H}), 3.65(\mathrm{t}, J$ $=6.2 \mathrm{~Hz}, 2 \mathrm{H}), 2.57(\mathrm{td}, J=6.9,4.4 \mathrm{~Hz}, 2 \mathrm{H}), 2.10-2.02(\mathrm{~m}, 2 \mathrm{H}), 1.37(\mathrm{t}, J=7.1 \mathrm{~Hz}, 6 \mathrm{H}) .{ }^{13} \mathrm{C} \mathrm{NMR}(75 \mathrm{MHz}$, $\left.\mathrm{CDCl}_{3}\right) \delta 100.9(\mathrm{~d}, J=53.0 \mathrm{~Hz}), 71.6(\mathrm{~d}, J=302.4 \mathrm{~Hz}), 63.2(\mathrm{~d}, J=5.5 \mathrm{~Hz}), 43.2,30.2(\mathrm{~d}, J=2.3 \mathrm{~Hz}), 16.7(\mathrm{~d}, J$ $=4.6 \mathrm{~Hz}), 16.1(\mathrm{~d}, J=7.0 \mathrm{~Hz}) \cdot{ }^{31} \mathrm{P}$ NMR $\left(121 \mathrm{MHz}, \mathrm{CDCl}_{3}\right) \delta-6.7 \mathrm{ppm} . \mathrm{MS}: m / z=238\left(\mathrm{M}^{+}, 1 \%\right), 185(10), 183$ (33), 176 (33), 175 (23), 165 (10), 161 (10), 149 (12), 148 (100), 147 (46), 133 (10), 129 (16), 120 (14), 93 (21), 91 (10), 83 (10), 81 (11), 66 (11), 65 (45). HRMS (EI) calcd for $\mathrm{C}_{9} \mathrm{H}_{16} \mathrm{ClO}_{3} \mathrm{P} 238.0526$, found 238.0531.

Diethyl (5-cyanopent-1-yn-1-yl)phosphonate (2j): Pale yellow oil. 91.6 mg, 80\% yield. IR (neat): 2978, 2929, 2896, 2242, 2210, 1254, 1041, $967 \mathrm{~cm}^{-1} .{ }^{1} \mathrm{H}$ NMR (300 MHz, $\left.\mathrm{CDCl}_{3}\right) \delta 4.19-4.06(\mathrm{~m}, 4 \mathrm{H}), 2.57-2.42(\mathrm{~m}, 4 \mathrm{H})$, $1.99-1.86(\mathrm{~m}, 2 \mathrm{H}), 1.34(\mathrm{t}, J=7.1 \mathrm{~Hz}, 6 \mathrm{H}) .{ }^{13} \mathrm{C} \mathrm{NMR}\left(75 \mathrm{MHz}, \mathrm{CDCl}_{3}\right) \delta 118.5,99.5(\mathrm{~d}, J=52.7 \mathrm{~Hz}), 72.6(\mathrm{~d}$, $J=301.5 \mathrm{~Hz}), 63.3(\mathrm{~d}, J=5.6 \mathrm{~Hz}), 23.5(\mathrm{~d}, J=2.4 \mathrm{~Hz}), 18.3(\mathrm{~d}, J=4.5 \mathrm{~Hz}), 16.2(\mathrm{~d}, J=7.2 \mathrm{~Hz}), 16.1(\mathrm{~d}, J=7.0$ Hz). ${ }^{31} \mathrm{P}$ NMR (121 MHz, $\left.\mathrm{CDCl}_{3}\right) \delta-7.1 \mathrm{ppm} . \mathrm{MS}: m / z=229\left(\mathrm{M}^{+}, 1 \%\right), 185$ (11), 183 (100), 157 (14), 156 (36), 155 (21), 144 (47), 143 (98), 142 (30), 131 (17), 130 (52), 129 (21), 128 (20), 127 (21), 118 (16), 117 (62), 116 (60), 115 (99), 104 (55), 103 (76), 102 (24), 91 (53), 90 (19), 89 (63), 78 (27), 77 (68), 76 (21), 75 (21), 74 (18), 65 (15), 64 (13), 63 (48), 62 (14), 51 (24), 50 (16). HRMS (EI) calcd for $\mathrm{C}_{10} \mathrm{H}_{16} \mathrm{NO}_{3} \mathrm{P} 229.0868$, found 229.0875.

Diethyl (3-hydroxyprop-1-yn-1-yl)phosphonate (2k) ${ }^{22}$ : Pale yellow oil. $61.5 \mathrm{mg}, 64 \%$ yield. ${ }^{1} \mathrm{H}$ NMR (300 $\left.\mathrm{MHz} \mathrm{CDCl}_{3}\right) \delta 4.84(\mathrm{~s}, 1 \mathrm{H}), 4.34(\mathrm{~d}, J=3.8 \mathrm{~Hz}, 2 \mathrm{H}), 4.20-4.10(\mathrm{~m}, 4 \mathrm{H}), 1.35(\mathrm{t}, J=7.1 \mathrm{~Hz}, 6 \mathrm{H}) .{ }^{13} \mathrm{C} \mathrm{NMR}(75$ $\left.\mathrm{MHz}, \mathrm{CDCl}_{3}\right) \delta 100.4(\mathrm{~d}, J=50.6 \mathrm{~Hz}), 74.1(\mathrm{~d}, J=298.7 \mathrm{~Hz}), 63.6(\mathrm{~d}, J=5.6 \mathrm{~Hz}), 50.6(\mathrm{~d}, J=4.4 \mathrm{~Hz}), 16.1(\mathrm{~d}, J$ $=7.1 \mathrm{~Hz}) .{ }^{31} \mathrm{P}$ NMR $\left(121 \mathrm{MHz}, \mathrm{CDCl}_{3}\right) \delta-6.8 \mathrm{ppm}$.

Diethyl (4-hydroxybut-1-yn-1-yl)phosphonate (2l): Yellow oil. 74.2 mg, 72\% yield. IR (neat): 3399, 2986, 2933, 2908, 2210, 1245, 1164, 1029, $980 \mathrm{~cm}^{-1} .{ }^{1} \mathrm{H}$ NMR (300 MHz, $\left.\mathrm{CDCl}_{3}\right) \delta 4.85(\mathrm{~s}, 1 \mathrm{H}), 4.22-4.07(\mathrm{~m}, 4 \mathrm{H})$, $3.79(\mathrm{t}, J=6.5 \mathrm{~Hz}, 2 \mathrm{H}), 2.61(\mathrm{td}, J=6.4,4.6 \mathrm{~Hz}, 2 \mathrm{H}), 1.37(\mathrm{t}, J=7.2 \mathrm{~Hz}, 6 \mathrm{H}) .{ }^{13} \mathrm{C} \mathrm{NMR}\left(75 \mathrm{MHz}, \mathrm{CDCl}_{3}\right) \delta$ $101.1(\mathrm{~d}, J=53.2 \mathrm{~Hz}), 71.4(\mathrm{~d}, J=302.8 \mathrm{~Hz}), 63.3(\mathrm{~d}, J=5.5 \mathrm{~Hz}), 59.8(\mathrm{~d}, J=2.5 \mathrm{~Hz}), 23.6(\mathrm{~d}, J=4.5 \mathrm{~Hz}), 16.1$ $(\mathrm{d}, J=7.1 \mathrm{~Hz}){ }^{31} \mathrm{P}$ NMR $\left(121 \mathrm{MHz}, \mathrm{CDCl}_{3}\right) \delta-6.3 \mathrm{ppm} . \mathrm{MS}: m / z=206\left(\mathrm{M}^{+}, 1 \%\right), 176(53), 148(45), 147$ (25), 
133 (23), 121 (13), 120 (100), 115 (12), 102 (21), 81 (10), 65 (13). HRMS (EI) calcd for $\mathrm{C}_{8} \mathrm{H}_{15} \mathrm{O}_{4} \mathrm{P}$ 206.0708, found 206.0711 .

Diethyl 3-(tetrahydro-2H-pyran-2-yl)oxy)prop-1-yn-1-ylphosphonate (2m): Pale yellow oil. 109.1 mg, 79\% yield. IR (neat): 2949, 2210, 1270, 1123, 1033, $976 \mathrm{~cm}^{-1} .{ }^{1} \mathrm{H}$ NMR (300 MHz, $\left.\mathrm{CDCl}_{3}\right) \delta 4.79(\mathrm{t}, J=2.8 \mathrm{~Hz}, 1 \mathrm{H})$, $4.36(\mathrm{~d}, J=3.8 \mathrm{~Hz}, 2 \mathrm{H}), 4.21-4.12(\mathrm{~m}, 4 \mathrm{H}), 3.86-3.76(\mathrm{~m}, 1 \mathrm{H}), 3.58-3.49(\mathrm{~m}, 1 \mathrm{H}), 1.81-1.51(\mathrm{~m}, 6 \mathrm{H}), 1.37$ $(\mathrm{t}, J=7.0 \mathrm{~Hz}, 6 \mathrm{H}) .{ }^{13} \mathrm{C} \mathrm{NMR}\left(75 \mathrm{MHz}, \mathrm{CDCl}_{3}\right) \delta 97.4,96.9(\mathrm{~d}, J=50.3 \mathrm{~Hz}), 75.8(\mathrm{~d}, J=295.5 \mathrm{~Hz}), 63.4(\mathrm{~d}, J=$ $5.5 \mathrm{~Hz}), 62.2,54.0(\mathrm{~d}, J=4.4 \mathrm{~Hz}), 30.2,25.4,18.9,16.2(\mathrm{~d}, J=7.0 \mathrm{~Hz}) .{ }^{31} \mathrm{P}$ NMR $\left(121 \mathrm{MHz}, \mathrm{CDCl}_{3}\right) \delta-7.3 \mathrm{ppm}$. MS: $m / z=276\left(\mathrm{M}^{+}, 1 \%\right), 275$ (12), 221 (81), 203 (18), 193 (52), 192 (20), 191 (28), 177 (15), 165 (100), 163 (18), 162 (12), 149 (11), 148 (48), 147 (43), 146 (25), 137 (21), 136 (16), 135 (26), 134 (13), 121 (13), 120 (43), 119 (29), 107 (10), 103 (37), 102 (18), 85 (18), 84 (15), 83 (14), 82 (10), 81 (14), 65 (18), 56 (10), 55 (44). HRMS (EI) calcd for $\mathrm{C}_{12} \mathrm{H}_{21} \mathrm{O}_{5} \mathrm{P} 276.1127$, found 276.1130 .

Diethyl nona-1,8-diyn-1-ylphosphonate (2n): Colorless oil. $87.1 \mathrm{mg}, 68 \%$ yield. IR (neat): 3301, 2990, 2937, 2855, 2206, 1258, 1045, $967 \mathrm{~cm}^{-1} .{ }^{1} \mathrm{H}$ NMR $\left(300 \mathrm{MHz}, \mathrm{CDCl}_{3}\right) \delta 4.22-4.06(\mathrm{~m}, 4 \mathrm{H}), 2.42-2.32(\mathrm{~m}, 2 \mathrm{H}), 2.26$ $-2.15(\mathrm{~m}, 2 \mathrm{H}), 1.96(\mathrm{t}, J=2.6 \mathrm{~Hz}, 1 \mathrm{H}), 1.66-1.50(\mathrm{~m}, 6 \mathrm{H}), 1.37(\mathrm{t}, J=7.2 \mathrm{~Hz}, 6 \mathrm{H}) .{ }^{13} \mathrm{C} \mathrm{NMR}(75 \mathrm{MHz}$, $\left.\mathrm{CDCl}_{3}\right) \delta 103.0(\mathrm{~d}, J=53.3 \mathrm{~Hz}), 84.1,70.5(\mathrm{~d}, J=304.1 \mathrm{~Hz}), 68.6,63.1(\mathrm{~d}, J=5.5 \mathrm{~Hz}), 27.9,27.8,27.0(\mathrm{~d}, J=$ $2.2 \mathrm{~Hz}), 19.2(\mathrm{~d}, J=4.5 \mathrm{~Hz}), 18.2,16.1(\mathrm{~d}, J=7.1 \mathrm{~Hz}) .{ }^{31} \mathrm{P}$ NMR $\left(121 \mathrm{MHz}, \mathrm{CDCl}_{3}\right) \delta-6.3 \mathrm{ppm} . \mathrm{MS}: m / z=256$ $\left(\mathrm{M}^{+}, 1 \%\right), 207$ (10), 200 (31), 199 (32), 185 (12), 172 (24), 171 (10), 161 (12), 148 (19), 147 (21), 145 (12), 135 (10), 133 (21), 131 (24), 121 (11), 120 (39), 119 (31), 118 (60), 117 (100), 115 (20), 105 (17), 103 (12), 102 (12), 93 (10), 92 (11), 91 (57), 81 (16), 79 (30), 77 (19), 65 (21), 53 (10). HRMS (EI) calcd for $\mathrm{C}_{13} \mathrm{H}_{21} \mathrm{O}_{3} \mathrm{P} 256.1228$, found 256.1231 .

Diethyl (3-oxobut-1-yn-1-yl)phosphonate (2o): Yellow oil. 96.9 mg, 95\% yield. IR (neat): 2990, 2921, 2839, 1687, 1266, 1204, 1021, 980, $857 \mathrm{~cm}^{-1} .{ }^{1} \mathrm{H}$ NMR (300 MHz, $\left.\mathrm{CDCl}_{3}\right) \delta 4.29-4.15(\mathrm{~m}, 4 \mathrm{H}), 2.43(\mathrm{~s}, 3 \mathrm{H}), 1.40(\mathrm{t}, J$ $=7.1 \mathrm{~Hz}, 6 \mathrm{H}) .{ }^{13} \mathrm{C} \mathrm{NMR}\left(75 \mathrm{MHz}, \mathrm{CDCl}_{3}\right) \delta 182.5(\mathrm{~d}, J=4.3 \mathrm{~Hz}), 92.7(\mathrm{~d}, J=43.0 \mathrm{~Hz}), 77.8(\mathrm{~d}, J=278.1 \mathrm{~Hz})$, $64.2(\mathrm{~d}, J=5.7 \mathrm{~Hz}), 32.5,16.2(\mathrm{~d}, J=6.8 \mathrm{~Hz}) .{ }^{31} \mathrm{P} \mathrm{NMR}\left(121 \mathrm{MHz}, \mathrm{CDCl}_{3}\right) \delta-9.1 \mathrm{ppm} . \mathrm{MS}: m / z=204\left(\mathrm{M}^{+}, 1 \%\right)$, 189 (31), 177 (18), 175 (13), 161 (71), 159 (30), 149 (81), 148 (49), 147 (39), 135 (25), 134 (100), 133 (88), 131 (14), 131 (67), 121 (11), 117 (13), 116 (11), 115 (20), 109 (15), 107 (47), 99 (10), 96 (11), 95 (30), 93 (21), 91 (14), 89 (59), 85 (11), 81 (27), 79 (11), 78 (13), 69 (10), 65 (46), 53 (44). HRMS (EI) calcd for $\mathrm{C}_{8} \mathrm{H}_{13} \mathrm{O}_{4} \mathrm{P}$ 204.0551, found 204.0555. 
Methyl 3-(diethoxyphosphoryl)propiolate (2p): Pale yellow oil. 106.7 mg, 97\% yield. IR (neat): 2990, 2962, 2132, 1732, 1433, 1282, 1164, 1045, 984, $882 \mathrm{~cm}^{-1} .{ }^{1} \mathrm{H}$ NMR (300 MHz, $\left.\mathrm{CDCl}_{3}\right) \delta 4.29-4.13(\mathrm{~m}, 4 \mathrm{H}), 3.83(\mathrm{~s}$, $3 \mathrm{H}), 1.38(\mathrm{t}, J=7.1 \mathrm{~Hz}, 6 \mathrm{H}) .{ }^{13} \mathrm{C} \mathrm{NMR}\left(75 \mathrm{MHz}, \mathrm{CDCl}_{3}\right) \delta 152.1(\mathrm{~d}, J=5.9 \mathrm{~Hz}), 86.5(\mathrm{~d}, J=46.6 \mathrm{~Hz}), 75.5(\mathrm{~d}, J$ $=279.1 \mathrm{~Hz}), 64.3(\mathrm{~d}, J=5.7 \mathrm{~Hz}), 53.6,16.2(\mathrm{~d}, J=6.8 \mathrm{~Hz}) .{ }^{31} \mathrm{P}$ NMR $\left(121 \mathrm{MHz}, \mathrm{CDCl}_{3}\right) \delta-9.8 \mathrm{ppm} . \mathrm{MS}: m / z=$ $220\left(\mathrm{M}^{+}, 1 \%\right), 193$ (13), 189 (47), 177 (13), 175 (13), 165 (58), 162 (16), 161 (33), 160 (21), 147 (56), 135 (83), 134 (82), 133 (100), 132 (14), 118 (13), 116 (16), 115 (25), 81 (24), 80 (24), 79 (39), 69 (11), 65 (32), 53 (32). HRMS (EI) calcd for $\mathrm{C}_{8} \mathrm{H}_{13} \mathrm{O}_{5} \mathrm{P} 220.0501$, found 220.0508 .

(E)-Diethyl (5-methoxy-3-methylpent-3-en-1-yn-1-yl)phosphonate (2q): Yellow oil. 116.9 mg, 95\% yield. IR (neat): 2982, 2933, 2908, 1650, 1474, 1249, 1168, 1029, 976, $861 \mathrm{~cm}^{-1} .{ }^{1} \mathrm{H} \mathrm{NMR}\left(300 \mathrm{MHz}, \mathrm{CDCl}_{3}\right) \delta 6.17(\mathrm{t}, J=$ $6.2 \mathrm{~Hz}, 1 \mathrm{H}), 4.17-4.03(\mathrm{~m}, 4 \mathrm{H}), 3.97(\mathrm{~d}, J=6.3 \mathrm{~Hz}, 2 \mathrm{H}), 3.28(\mathrm{~s}, 3 \mathrm{H}), 1.78(\mathrm{~s}, 3 \mathrm{H}), 1.31(\mathrm{t}, J=7.1 \mathrm{~Hz}, 6 \mathrm{H}) .{ }^{13} \mathrm{C}$ NMR $\left(75 \mathrm{MHz}, \mathrm{CDCl}_{3}\right) \delta 140.2(\mathrm{~d}, J=3.4 \mathrm{~Hz}), 118.4(\mathrm{~d}, J=5.9 \mathrm{~Hz}), 101.1(\mathrm{~d}, J=52.1 \mathrm{~Hz}), 76.2(\mathrm{~d}, J=300.3$ $\mathrm{Hz}), 68.6,63.3(\mathrm{~d}, J=5.5 \mathrm{~Hz}), 58.6,16.8(\mathrm{~d}, J=1.8 \mathrm{~Hz}), 16.2(\mathrm{~d}, J=7.0 \mathrm{~Hz}) .{ }^{31} \mathrm{P} \mathrm{NMR}\left(121 \mathrm{MHz}, \mathrm{CDCl}_{3}\right) \delta-5.9$ ppm. MS: $m / z=246\left(\mathrm{M}^{+}, 68 \%\right), 231$ (55), 218 (16), 217 (28), 203 (38), 190 (12), 189 (29), 175 (100), 171 (20), 161 (22), 160 (11), 159 (20), 157 (96), 147 (14), 143 (12), 141 (21), 129 (17), 109 (28), 95 (34), 91 (10), 81 (14), 79 (35), 78 (17), 77 (39), 67 (19), 66 (10), 65 (30), 53 (10), 51 (15). HRMS (EI) calcd for $\mathrm{C}_{11} \mathrm{H}_{19} \mathrm{O}_{4} \mathrm{P} 246.1021$, found 246.1024 .

Diethyl (cyclohex-1-en-1-yl)ethynylphosphonate (2r): Pale yellow oil. 112.6 mg, 93\% yield. IR (neat): 2984, 2937, 2868, 2173, 1687, 1258, 1160, 1037, $984 \mathrm{~cm}^{-1} .{ }^{1} \mathrm{H}$ NMR (300 MHz, $\left.\mathrm{CDCl}_{3}\right) \delta 6.42(\mathrm{~s}, 1 \mathrm{H}), 4.21-4.07(\mathrm{~m}$, 4H), $2.15-2.10(\mathrm{~m}, 4 \mathrm{H}), 1.65-1.55(\mathrm{~m}, 4 \mathrm{H}), 1.35(\mathrm{t}, J=7.1 \mathrm{~Hz}, 6 \mathrm{H}) .{ }^{13} \mathrm{C} \mathrm{NMR}\left(75 \mathrm{MHz}, \mathrm{CDCl}_{3}\right) \delta 142.0(\mathrm{~d}, J$ $=3.3 \mathrm{~Hz}), 118.5(\mathrm{~d}, J=5.9 \mathrm{~Hz}), 101.6(\mathrm{~d}, J=52.8 \mathrm{~Hz}), 75.7(\mathrm{~d}, J=302.8 \mathrm{~Hz}), 63.1(\mathrm{~d}, J=5.4 \mathrm{~Hz}), 28.0(\mathrm{~d}, J=$ $1.6 \mathrm{~Hz}), 26.0,21.9,21.1,16.2(\mathrm{~d}, J=7.1 \mathrm{~Hz}) .{ }^{31} \mathrm{P} \mathrm{NMR}\left(121 \mathrm{MHz}, \mathrm{CDCl}_{3}\right) \delta-5.2 \mathrm{ppm} . \mathrm{MS}: m / z=242\left(\mathrm{M}^{+}\right.$, 64\%), 241 (27), 214 (28), 213 (45), 207 (30), 199 (16), 197 (22), 187 (12), 186 (76), 185 (72), 171 (11), 170 (20), 169 (42), 159 (10), 158 (11), 150 (16), 149 (22), 145 (15), 135 (17), 134 (25), 133 (40), 132 (35), 131 (34), 122 (13), 121 (13), 120 (16), 119 (15), 117 (44), 107 (21), 106 (98), 105 (91), 104 (70), 103 (69), 102 (12), 93 (14), 92 (15), 91 (100), 89 (12), 81 (12), 79 (36), 78 (48), 77 (66), 65 (31), 63 (11), 55 (13), 51 (13). HRMS (EI) calcd for $\mathrm{C}_{12} \mathrm{H}_{19} \mathrm{O}_{3} \mathrm{P} 242.1072$, found 242.1075.

Diethyl (3-(1,3-dioxoisoindolin-2-yl)prop-1-yn-1-yl)phosphonate (2s): White solid; mp 76.0 - $78.2{ }^{\circ} \mathrm{C} .141 .3$ mg, 88\% yield. IR (KBr): 2990, 2962, 2921, 2218, 1715, 1609, 1425, 1258, 1119, 1045, 1021, 963, 726, 66514 
$\mathrm{cm}^{-1} .{ }^{1} \mathrm{H}$ NMR $\left(300 \mathrm{MHz}, \mathrm{CDCl}_{3}\right) \delta 7.92-7.86(\mathrm{~m}, 2 \mathrm{H}), 7.80-7.74(\mathrm{~m}, 2 \mathrm{H}), 4.58(\mathrm{~d}, J=3.9 \mathrm{~Hz}, 2 \mathrm{H}), 4.23-$ $4.07(\mathrm{~m}, 4 \mathrm{H}), 1.36(\mathrm{t}, J=7.1 \mathrm{~Hz}, 6 \mathrm{H}) .{ }^{13} \mathrm{C} \mathrm{NMR}\left(75 \mathrm{MHz}, \mathrm{CDCl}_{3}\right) \delta 166.5,134.5,131.8,123.8,93.5(\mathrm{~d}, J=50.6$ $\mathrm{Hz}), 73.5(\mathrm{~d}, J=293.5 \mathrm{~Hz}), 63.5(\mathrm{~d}, J=5.5 \mathrm{~Hz}), 27.3(\mathrm{~d}, J=4.6 \mathrm{~Hz}), 16.1(\mathrm{~d}, J=7.0 \mathrm{~Hz}) .{ }^{31} \mathrm{P}$ NMR $(121 \mathrm{MHz}$, $\left.\mathrm{CDCl}_{3}\right) \delta-7.8$ ppm. MS: $m / z=321\left(\mathrm{M}^{+}, 1 \%\right), 293$ (11), 292 (10), 276 (13), 265 (10), 248 (19), 214 (17), 213 (100), 212 (26), 186 (11), 185 (31), 184 (66), 161 (12), 160 (40), 157 (13), 156 (11), 148 (10), $146(34), 133$ (15), 130 (71), 129 (11), 119 (11), 105 (16), 104 (42), 102 (17), 77 (13), 76 (32). HRMS (EI) calcd for $\mathrm{C}_{15} \mathrm{H}_{16} \mathrm{NO}_{5} \mathrm{P}$ 321.0766, found 321.0772.

\section{Diethyl [(8R,9S,13S,14S,17S)-3,17-dihydroxy-13-methyl-7,8,9,11,12,13,14,15,16,17-decahydro-6H-} cyclopenta[a]phenanthren-17-yl]ethynylphosphonate (2t): Pale yellow solid; mp 79.5-82.0 ${ }^{\circ} \mathrm{C} .170 .7 \mathrm{mg}$, 79\% yield. IR (KBr): 3321, 2986, 2937, 2872, 2197, 1617, 1511, 1450, 1241, 1025, 980, $816 \mathrm{~cm}^{-1} .{ }^{1} \mathrm{H}$ NMR (300 $\left.\mathrm{MHz}, \mathrm{CDCl}_{3}\right) \delta 7.02(\mathrm{~d}, J=8.5 \mathrm{~Hz}, 1 \mathrm{H}), 6.67(\mathrm{dd}, J=8.5,2.4 \mathrm{~Hz}, 1 \mathrm{H}), 6.59(\mathrm{~d}, J=2.4 \mathrm{~Hz}, 1 \mathrm{H}), 4.27-4.14(\mathrm{~m}$, $4 \mathrm{H}), 2.82-2.69(\mathrm{~m}, 2 \mathrm{H}), 2.41-2.27(\mathrm{~m}, 1 \mathrm{H}), 2.20-2.10(\mathrm{~m}, 1 \mathrm{H}), 2.07-1.97(\mathrm{~m}, 2 \mathrm{H}), 1.94-1.83(\mathrm{~m}, 1 \mathrm{H})$, $1.82-1.68(\mathrm{~m}, 4 \mathrm{H}), 1.61-1.48(\mathrm{~m}, 1 \mathrm{H}), 1.45-1.33(\mathrm{~m}, 3 \mathrm{H}), 1.39(\mathrm{td}, J=7.0,2.8 \mathrm{~Hz}, 6 \mathrm{H}), 0.86(\mathrm{~s}, 3 \mathrm{H}) .{ }^{13} \mathrm{C}$ NMR $\left(75 \mathrm{MHz}, \mathrm{CDCl}_{3}\right) \delta 154.5,137.8,131.5,126.5,115.3,112.9,105.3(\mathrm{~d}, J=49.5 \mathrm{~Hz}), 80.3(\mathrm{~d}, J=3.8 \mathrm{~Hz})$, $75.3(\mathrm{~d}, J=314.0 \mathrm{~Hz}), 63.7(\mathrm{dd}, J=5.5,1.2 \mathrm{~Hz}), 50.2,48.0,43.3,39.4,38.7,31.1,29.7,27.4,26.4,23.1,16.3$ $(\mathrm{dd}, J=6.9,1.2 \mathrm{~Hz}), 12.8 .{ }^{31} \mathrm{P} \mathrm{NMR}\left(121 \mathrm{MHz}, \mathrm{CDCl}_{3}\right) \delta-6.2 \mathrm{ppm}$. (The signal corresponding to OH were not observed). MS: $m / z=432\left(\mathrm{M}^{+}, 1 \%\right), 271$ (20), 270 (100), 214 (10), 213 (21), $186(12), 185$ (34), 172 (27), 160 (16), 159 (17), 158 (12), 157 (15), 146 (31), 145 (18), 144 (10), 133 (12), 131 (11), 115 (12). HRMS (EI) calcd for $\mathrm{C}_{24} \mathrm{H}_{33} \mathrm{O}_{5} \mathrm{P} 432.2066$, found 432.2065 .

\section{Supporting Information.}

Copies of ${ }^{1} \mathrm{H}-,{ }^{13} \mathrm{C}$ - and ${ }^{31} \mathrm{P}-\mathrm{NMR}$ spectra of all alkynylphosphonate products. This material is available free of charge via the Internet at http://pubs.acs.org.

\section{Corresponding Author.}

E-mail: gradivoy@criba.edu.ar; ymoglie@uns.edu.ar

\section{Notes}

The authors declare no competing financial interest.

\section{ACKNOWLEDGMENT}


This work was generously supported by the Consejo Nacional de Investigaciones Científicas y Técnicas (CONICET, PIP-2011-11220100100268), Agencia Nacional de Promoción Científica y Tecnológica (ANPCyT, Préstamo BID PICT-2010-0669) and Universidad Nacional del Sur (UNS, PGI 24/Q044) from Argentina. V.G. thanks the CONICET for a postdoctoral fellowship.

\section{REFERENCES}

(1) (a) For a recent review, see: Demmer, C. S.; Krogsgaard-Larsen, N.; Bunch, L. Chem. Rev. 2011, $111,7981$.

(b) Debora G. Salomon, D. G.; Grioli, S. M.; Buschiazzo, M.; Mascaró, E.; Vitale, C.; Radivoy, G.; Perez, M.; Fall, Y.; Mesri, E. A.; Curino, A. C.; Facchinetti, M. M. ACS Med. Chem. Lett. 2011, 2, 503.

(2) (a) Gil, J. M.; Oh, D. Y. J. Org. Chem. 1999, 64, 2950; (b) Quntar, A. A.; Srebnik, M. Org. Lett. 2001, 3, 1379.

(c) Ruder, S. M.; Norwood, B. K. Tetrahedron Lett. 1994, 35, 3473. (d) Nishida, G.; Noguchi, K.; Hirano, M.; Tanaka, K. Angew. Chem., Int. Ed. 2007, 46, 3951. (e) Evano, G.; Coste, A.; Jouvin, K. Angew. Chem., Int. Ed., 2010, 49, 2840. (f) DeKorver, K. A.; Li, H.; Lohse, A. G.; Hayashi, R.; Lu, Z.; Zhang, Y.; Hsung, R. P. Chem. Rev., 2010, 110, 5064. (g) Wang, X.-N.; He, S.-Z.; Fang, L.; Yeom, H.-S.; Kedrowski, B. L.; Hsung, R. P. Acc. Chem. Res. 2014, 47, 560 .

(3) Krishna, H.; Caruthers, M. H. J. Am. Chem. Soc. 2012, 134, 11618.

(4) Iorga, B.; Eymery, F.; Carmichael, D.; Savignac, P. Eur. J. Org. Chem. 2000, 3103, and references cited therein.

(5) (a) Wang, Y.; Gan,J.; Liu, L.; Yuan, H.; Gao, Y.; Liu, Y.; Zhao, Y. J. Org. Chem. 2014, 79, 3678. (b) Lera, M.; Hayes, C. J. Org. Lett. 2000, 2, 3873.

(6) Hu, J.; Zhao, N.; Yang, B.; Wang, G.; Guo, L.-N.; Liang, Y.-M.; Yang, S.-D. Chem. Eur. J. 2011, $17,5516$.

(7) Jouvin, K.; Heimburger, J.; Evano, G. Chem. Sci. 2012, 3, 756.

(8) Yatsumonji, Y.; Ogata, A.; Tsubouchi, A.; Takeda, T. Tetrahdron Lett. 2008, 49, 2265.

(9) Lecerclé, D.; Mothes, C.; Taran, F. Synth. Commun. 2007, 37, 1301.

(10) Li, X.; Sun, S.; Yang, F.; Kang, J.; Wu, Y.; Wu, Y. Org. Biomol. Chem. 2015, 13, 2432.

(11) Gao, Y.; Wang, G.; Chen, L.; Xu, P.; Zhao, Y.; Zhou, Y.; Han, L.-B. J. Amer. Chem. Soc. 2009, 131, 7956.

(12) Liu, P.; Yang, J.; Li, P.; Wang, L. Appl. Organometal. Chem. 2011, 5, 830. 
(13) (a) Yang, J.; Chen, T.; Zhou, Y.; Shuangfeng, Y.; Han, L.-B. Chem. Commun. 2015, 51, 3549. (b) Yang, J.; Chen, T.; Zhou, Y.; Shuangfeng, Y.; Han, L.-B. Organometllics 2015, 34, 5095.

(14) (a) Alonso, F.; Moglie, Y.; Radivoy, G.; Yus, M. Green Chem. 2012, 14, 2699. (b) Alonso, F.; Moglie, Y. Curr. Green Chem. 2014, 1, 87. (c) Gutierrez,V.; Mascaró, E.; Alonso, F.; Moglie, Y.; Radivoy, G. RSC Adv. 2015, 5,65739 .

(15) For a review on aerobic copper-catalyzed organic reactions, see: Allen, S. E.; Walvord, R. R.; PadillaSalinas, R.; Kozlowski, M. C. Chem. Rev. 2013, 113, 6234.

(16) (a) For the observation of $\mathrm{Cu}(\mathrm{I}) / \mathrm{Cu}(\mathrm{III})$ redox steps in cross-coupling reactions, see: Casitas, A.; King, A. E.; Parella, T.; Costas, M.; Stahl, S. S.; Ribas, X. Chem. Sci. 2010, 1, 326. See also: (b) Alonso, F.; Moglie, Y.; Radivoy, G.; Yus, M. Synlett 2012, 23, 2179.

(17) Dizière, R.; Savignac, P. Tetrahedron Lett. 1996, 37, 1783.

(18) Lera, M; Hayes, C. J. Org. Lett. 2000, 2, 3873.

(19) Li X.; Sun, S.; Yang, F.; Kang, J.; Wu, Y.; Wu, Y. Org. Biomol. Chem. 2015, 13 (8), 2432.

(20) Wang, Y.; Gan, J.; Liu, L.; Yuan, H.; Gao, Y.; Liu, Y.; Zhao, Y. J. Org. Chem. 2014, 79, 3678.

(21) Chen, C. C.; Waser, J. Chem. Commun. 2014, 50, 12923.

(22) Cockburn, N.; Karimi, E.; Tam, W. J. Org. Chem. 2009, 74, 5762. 